\title{
Estimation of Serum Copper in Post-menopausal Women Attending in a Tertiary Care Hospital, Bangladesh
}

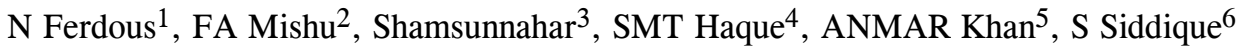

\begin{abstract}
Background: Nutritional needs change throughout the various stages of life. While overall caloric needs tend to decrease with age, the requirements for individual micronutrients do not decrease. In fact, the needs for some micronutrients actually increase with age. The risk of nutritional disturbances mainly trace elements deficiencies is high during postmenopause causing or intensifying serious clinical symptoms.

Methods: This cross sectional study was carried out in the Department of Biochemistry, Mymensingh Medical College in collaboration with the Department of Obstetrics and Gynecology, Mymensingh Medical College Hospital during the period from July 2013 to June 2014. A total of 100 subjects were included in this study. Among them 50 apperantly healthy postmenopausal women (Case) aged between 45-60 years were considered as case and another 50 regular menstruating premenopausal women (Control) aged between 30-40 years were selected as control.
\end{abstract}

Results: The mean age of cases and control groups was $50.5 \pm 2.3$ years and $36.5 \pm 4.4$ years respectively. In this study we found Serum Copper levels in cases and controls were $115.36 \pm 8.836 \mu \mathrm{g} / \mathrm{dl}$ and $90.58 \pm 6.315 \mu \mathrm{g} / \mathrm{dl}$ respectively had $(\mathrm{p}<0.01)$ significant difference.

Conclusion: Serum Copper level was significantly increased in postmenopausal group in comparison to premenopausal women $(\mathrm{p}<0.01)$. Estimation of serum $\mathrm{Cu}$ level might be incorporated in every postmenopausal women for prevention of complications.

Key Note: micronutrients, Post menopausal.

\section{Introduction}

Menopause is defined as, the permanent cessation of menstruation for a period of longer than one year. Natural menopause is a gradual process. Unless brought about by surgery, chemotherapy or other

factors, natural or physiologic menopause occurs in most women between the ages of 47-55 years and the average age of natural menopause is 51 years. $^{1}$ Although menopause is a natural biologic process

\footnotetext{
${ }^{1 *}$ Dr. Nadia Ferdous, Assistant Professor of Biochemistry, Tairunnessa memorial Medical College, Dhaka, e-mail Address: shuchinf@gmail.com

${ }^{2}$ Dr. Farzana Akonjee Mishu, Assistant Professor, Department of Physiology and Molecular Biology, Bangladesh Institute of Research\& Rehabilitation In Diabetes, Endocrine \& Metabolic Disorders (BIRDEM) Dhaka, Bangladesh

${ }^{3}$ Dr.Shamsunnahar, Assistant Professor of Biochemistry, president Abdul Hamid Medical College Kishorgonj

${ }^{4}$ Dr. Syed Md. Tanjilul Haque, Associate Professor,Department of Forensic Medicine, Anwer Khan Modern Medical College, Dhaka

${ }^{5}$ Dr. A.N.M.Ashikur Rahman Khan, Medical Officer, Institutional Affiliation, Department of NIDCH

${ }^{6}$ Dr. Sohana Siddique, Associate Professor ,Department of Gynae and Obstetrics International Medical College and Hospital, Tongi

*Corresponding Author

Date of submission: 26.02.2019 Date of acceptance: 27.03.2019
}

AKMMC J 2019; 10(2) : 110-113 
occurring in the body due to changing hormone levels, across the life span of a woman, it probably has the greatest impact on health. ${ }^{2}$ Women face various physiological, psychological and biochemical changes during this period. The most common symptom reported by postmenopausal women is - hot flush or night sweats $(80 \%)$. The other symptoms may include tiredness, mood swings, insomnia, mental exhaustion, joint and muscular discomfort, irritability, headache, dryness of vagina, loss of libido, urinary incontinence etc. ${ }^{3}$ In this period of life the risk of osteoporosis, cardiovascular diseases, arterial hypertension, impairment of glucose metabolism, breast cancer and degenerative cognition diseases rises. The risk of nutritional disturbances, particularly trace elements and vitamin deficiencies is also high during this period. ${ }^{4,5}$ It is the time when steroid hormone deficiency is often accompanied by essential mineral deficiencies causing or intensifying the clinical symptoms.

Trace elements are essential co factors for enzymes, involved in the synthesis of various bone matrix constituents. They are also involved in the interaction of many enzyme reactions necessary for the transmission of nerve impulses, temperature regulation, detoxification, energy production etc. ${ }^{6}$ Nutritional needs change throughout the various stages of life. While overall caloric needs tend to decrease with age, the requirements for individual micronutrients (vitamins and nutritionally-essential minerals) do not decrease. In fact, the needs for some micronutrients, actually increase with age. Adults older than 50 years, require higher intakes of these micronutrients. ${ }^{7,8}$ Alteration of the levels of trace elements results in altered immune functions, increased oxidative stress, impaired cognitive functions, glucose intolerance, osteoporosis etc in postmenopausal age group. ${ }^{9}$

Copper is an essential trace element that is vital to health of all living things (humans, animals and microorganisms). In humans, copper is essential for the proper functioning of organs and metabolic processes. It is an essential co-factor for a number of enzymes involved in metabolic reactions, angiogenesis, oxygen transport and anti-oxidant production including catalase, superoxide dismutase (SOD) and cytochrome oxidase. ${ }^{7,8}$ Copper induces a low bone turnover by suppression of the osteoblastic and the osteoclastic functions. ${ }^{10} \mathrm{~A}$ decrease in serum copper levels observed in postmenopausal women which may be due to advanced age. ${ }^{4,11}$ Copper deficiency can also results from malnutrition, malabsorption, or excessive zinc intake. ${ }^{7}$ Copper imbalance has been linked to impaired immune function, bone demineralization and increased risk of cardiovascular and neurodegenerative diseases. ${ }^{8,12,13}$ However, the use of more precise indicators of nutritional copper status needs to be considered for future research. ${ }^{14}$

\section{Materials and Methods}

The study was carried out at Mymensingh Medical College Hospital from July 2013 to June 2014 to evaluate the serum level of Copper in 50 apparently healthy postmenopausal (45-60 years) women and compared with 50 healthy premenopausal (30-40 years) women. Pre menopausal women were treated as control group. Post-menopausal women had at least one year of amenorrhea. None had received estrogen therapy or any supportive treatment for menopausal symptoms for at least 6months prior to study. The study protocol was approved by the institutional review committee and written informed consent was obtained from all the participants prior to their enrolment into this study.

Study population and collection of samples: Postmenopausal women, who were attending the outpatient department of Obstetrics and Gynecology of Mymensingh Medical College Hospital were enrolled by purposive sampling technique. Postmenopausal women with the previous history of diabetes, hypertension and other endocrine disorders, hormone replacement therapy, surgical menopause women were excluded from the study. Data were collected in a predesigned data collection sheet. The variables included were - age, height, weight, family history. About $3 \mathrm{ml}$ of blood was collected aseptically with venipuncture from all participants for estimation of serum $\mathrm{Cu}$ levels. 
Estimation serum $\mathrm{Cu}$ : Serum $\mathrm{Cu}$ was determined by colorimetric method by 3, 5-DiBr-PAESA method and The results were analyzed statistically and values were expressed as mean \pm SD. The level of significance was determined by employing Student's unpaired $t$ test. Only when the $\mathrm{p}$ value was less than 0.05 ; the difference between two groups were considered as statistically significant.

\section{Result}

In the present study, out of 100 subjects 50 apparently healthy postmenopausal women were selected as case and 50 premenopausal women as control. Different variables of the subjects were being analyzed and compared between case and control. Statistical significance of difference between two groups were evaluated by using Student's unpaired t-test. It was observed that mean Copper $115.36 \pm 8.84 \mu \mathrm{g} / \mathrm{dl}$ ) for cases and 90.58 $\pm 6.31(\mu \mathrm{g} / \mathrm{dl})$ for controls. The difference in mean serum Copper levels were highly significant

Table-1: Clinical and Biochemical Characteristics of the study subjects.

\begin{tabular}{lccc}
\hline Variables & $\begin{array}{c}\text { Mean } \pm \text { SD } \\
\text { (cases) }\end{array}$ & $\begin{array}{c}\text { Mean } \pm \text { SD } \\
\text { (controls) }\end{array}$ & P value \\
\hline Age & $50.5 \pm 2.3$ & $36.5 \pm 4.4$ & $<0.001$ \\
Height $(\mathrm{cm})$ & $149.21 \pm 10.12$ & $150.31 \pm 11.42$ & $>0.05$ \\
Weight $(\mathrm{kg})$ & $57.43 \pm 8.41$ & $58.46 \pm 7.39$ & $>0.05$ \\
Systolic BP $(\mathrm{mm} \mathrm{Hg})$ & $140 \pm 6.15$ & $130 \pm 5.38$ & $<0.001$ \\
Diastolic BP $(\mathrm{mmHg})$ & $85 \pm 2.44$ & $80 \pm 4.21$ & $<0.01$ \\
Copper $(\mu \mathrm{g} / \mathrm{dl})$ & $115.36 \pm 8.836$ & $90.58 \pm 6.315$ & $<0.01$ \\
\hline
\end{tabular}

\section{Discussion}

As a woman ages, her copper status may alter for several reasons. In this study, serum copper in both pre and postmenopausal women was estimated. A significant increase in serum copper level was observed in the postmenopausal women as compared to that in the premenopausal women, which agrees with the previous reports by Bureau, et al. 2002, ${ }^{12}$ Naveenta and Khushdeep, 2011.15 Although copper deficiency appears to be common in older women as a result of low dietary intake or reduced dietary absorption, excess of copper in body may cause depression, irritability, fatigue, nervousness, nausea, vomiting, digestive disorders, joint/muscle and bone pain, jaundice, premature aging, peripheral oedema, dizziness ${ }^{16}$ etc.

In older people decreased copper intake and absorption reduces the activity of the copperdependent enzyme lysyl oxidase, which is required for the maturation of collagen-a key element in the organic matrix of bone. Copper also induces a low bone turnover by suppression of the osteoblastic and osteoclastic function. ${ }^{10,18,19}$ Among the studies on copper and bone health, researchers found a definite correlation between copper and lower bone density and bone strength. It has been demonstrated that copper can have a positive impact on the spinal bone densities of postmenopausal women. ${ }^{17}$ According to some study serum copper is low in postmenopausal women in comparison to premenopausal age group. ${ }^{15}$ Some study state that, the administration of estrogen during hormone replacement therapy has shown an improvement in the serum copper level in postmenopausal women. ${ }^{4}$ While some study could not show any significant difference in serum copper among postmenopausal women while comparing to premenopausal women. ${ }^{9}$

Copper is nutritionally essential element needed for catalytic, structural and regulatory functions for all forms of life. ${ }^{18}$ The significance of copper in menopausal nutrition and public health was recognized relatively recently. Since then, trace elements insufficiency has been recognized by a number of experts as an important public health issue, especially in developing countries. ${ }^{10}$

\section{Conclusion}

As postmenopausal women are exposed to greater risk of serum biochemical changes and possibility of nutritional disturbances particularly trace elements and vitamin deficiencies and risk of age related diseases is very high during this period, these adverse changes in serum copper should be taken into consideration for early diagnosis and prevention of menopause related diseases. Dietary supplementation may be necessary especially where levels are significantly reduced. Therefore, further study should be done to find out the underlying 
mechanism of alteration of serum copper in post menopausal women.

\section{Conflict of interest: None.}

\section{References}

1. Padubidri, V.G., Daftari, S. N. 2004. "Bourne Shaw's Text Book of Gynaecology". 13 $3^{\text {th }}$ ed. Elsevier, New Delhi. pp. 56 - 58.

2. Baber, R. Phytoestrogens and post reproductive health. Maturitas, 2010; 66: 344-9

3. Rahman et al. Menopausal symptoms assessment among middle age women in Kushtia, Bangladesh. Bmc Research Notes 2011; 4: 188.

4. Bednarek - Tupikowska, G., Jodkowska, A., Antonowicz - Juchiniewicz, J. Zinc, Copper , Manganese and selenium status in pre and post menopausal women during sex hormone therapy. Adv Clin Exp Med 2010; 19(3): 337345.

5. Davis, S. R. Menopause: New therapies. MJA 2003; 178: 634-7.

6. Kirk, D. Fish, S. A. Medical management of osteoporosis. Am J Mang Care 2004; 10: 39-43

7. Stern Bonnie, R. "Essentiality and Toxicity In Copper Health Risk Assessment: Overview, Update And Regulatory Considerations". Journal of Toxicology and Environmental Health. Part A 2010; 73: 114-127.

8. Stern Bonnie, R. et al. "Copper and Health: Biochemistry, GeneticsHuman, and Strategies For Modeling Dose-Response Relationships". Journal of Toxicology and Environmental Health. Part B 2007; 10: 157-222.

9. Mutlu, M., Argun, M., Saraymen, R., et al. mgnesium, zinc and copper status in osteoporotic, osteopenic and normal postmenopausal women. The Journal of International Medical Research 2007; 35: 692-695.

10. Gur, A., Colpan, L., Nas, K., et al. The role of trace minerals in the pathogenesis of postmenopausal osteoporosis and a new effect of calcitonon. J Bone Miner Metab 2002; 2; 20: 39-43.
11. Ansar, S., Alhefdhi, T., Allem, A.M. Status of trace elements and antioxidants in premenopausal and post menopausal phase of life: A comparative Study. Int J Clin Exp Med 2015; 8(10): 19486-90.

12. Bureau, B., Anderson, RA., Arnaud, J., Raysiguier, $\mathrm{Y}$ et al. Trace mineral status in post menopausal women: impact of hormonal replacement therapy. J Trace Elem Med Biol. 2002; 16(1): 9-13.

13. Martin-Lagos, F., Navarro-Alarcon, M., TerresMartos, et al. Zinc and Copper concentrations in serum from Spanish women during pregnancy. Biol Trace Elem Res 1998; 61(1): 61-70.

14. Scheiber, Ivo; Dringen, Ralf, Mercer, et al. "Chapter 11. Copper: Effects of Deficiency and Overload". In Astrid Sigel, Helmut Sigel and Roland K. O. Sigel. Interrelations between Essential Metal Ions and Human Diseases. Metal Ions in Life Sciences 2013; 13 Springer: 359-387.

15. Naveenta Gupta, Khushdeep Singh Arora. The Status of Trace Elements after Menopause: A Comparative Study. Journal of Clinical and Diagnostic Research 2011; 5( 4): 795 - 97.

16. Spinazzi, M., De Lazzari, F., Tavolato, B., et al. "Myelo-optico-neuropathy in copper deficiency occurring after partial gastrectomy. Do small bowel bacterial overgrowth syndrome and occult zinc ingestion tip the balance?". J Neurol 2007; 254: 1012-17.

17. Saltman, P.D., Strause, L. G. The role of trace minerals in osteoporosis. J Am Coll 1993; 12: 384-389.

18. Nedrow, A., Miller, J., Walker, M., Nygren, P., et al. "Complementary and alternative therapies for the management of menopause-related symptoms: a systematic evidence review". Arch Intern Med 2006; 166(14): 1453-65.

19. Rowin, J., Lewis, S. L. Copper deficiency myeloneuropathy and pancytopenia secondary to overuse of zinc supplementation. J Neurol Neurosurg Psychiatry. 2005; 76(5): 750-751. (PubMed)

20. Smith, T., Contestabile, E. Executive summary: Canadian consensus on menopause and osteoporosis. J SOGC 2001; 23: 829-835. 